\title{
UNDERSTANDING INVASIVE, AND RAPID TRANSMITTING NATURE OF CORONA THROUGH (AAIR) AND BY (IS- Tn -IS) DNA PROBE ANALYSIS.
}

\author{
NITOSH BRAHMA,FIE ${ }^{1}$ \\ ${ }^{1}$ Institution of Engineers India
}

June 17, 2020

\begin{abstract}
Antiadherent Immune Response (AAIR) against serotype 026: EPEC (Enteropathogenic (invasive) Escherichia coli) a fatal diarrhoea was successful in Balb/c mice experiment by the author. IS(Insertion Sequence) represented by IS1,IS2,IS3,- . . . . IS10 flanking transpons ( Tn)" IS-TN-IS" and its illegitimate recombination was also studied curiously by the author to observe their spontaneous jumping, illegitimate recombination among DNA, chromosome and plasmids in Escherichia coli. Considering the fatality rate of corona as pandemic globally, the author has attempted to realise the possible application of AAIR and IS-Tn-IS mixed hybrid concept, in designing vaccine against corona, to prevent corona virus not to adhere in trachea and lung cells, to bring healthy life to mankind. IS-Tn-IS was studied in Maize by Barbara Mc Clintock, USA, Peter Starlinger and H.Saedler, Germany studied the same in bacteria.. In this paper the author describes the possible AAIR vaccine against corona. A mixture of combine strategy corona vaccine with IS-Tn-IS gene expression.
\end{abstract}

UNDERSTANDING INVASIVE, AND RAPID TRANSMITTING NATURE OF CORONA THROUGH (AAIR) AND BY (IS- Tn -IS) DNA PROBE ANALYSIS.

PROF.NITOSH KUMAR BRAHMA,FIE,

CONVENR CHEM.ENGG. DIV, WBSC/IEI,( THE INSTITUTION OF ENGINEERS,INDIA)

VISITING PROFESSOR IGE (INSTITUTE OF GENETIC ENGINEERING)-BADU ,KOLKATA.

EX-FACULTY IIT-KHARAGPUR, DEPT.OF CHE.ENGG, KHARAGPUR-721302, INDIA. profbrahma@gmail.com, $+919830285504 ;+919732686077$

Abstract:

Antiadherent Immune Response (AAIR) against serotype 026: EPEC (Enteropathogenic (invasive) Escherichia coli) a fatal diarrhoea was successful in Balb/c mice experiment by the author. IS(Insertion Sequence) represented by IS1,IS2,IS3,-..... IS10 flanking transposons ( Tn)"IS-TN-IS" and its illegitimate recombination was also studied curiously by the author to observe their spontaneous jumping, illegitimate recombination among DNA, chromosome and plasmids in Escherichia coli. Considering the fatality rate of corona as pandemic globally, the author has attempted to realize the possible application of AAIR and IS-Tn-IS mixed hybrid concept, in designing vaccine against corona, to prevent corona virus not to adhere in trachea 
and lung cells, to bring healthy life to mankind. IS-Tn-IS was studied in Maize by Barbara Mc Clintock, USA, Peter Starlinger and H.Saedler, Germany studied the same in bacteria.. In this paper the author describes the possible AAIR vaccine against corona. A mixture of combine strategy corona vaccine with IS-Tn-IS gene expression.

\section{Introduction.}

IS1 sequence is found 8 copies in Escherichia coli k-12. IS1.....IS10 are mainly existing as flanking gene in repeats and inverted repeats on both sides of transposable elements, characterised by Tn3 as Ampicillin, Tn5 as Chloramphenicol, and Tn10 as Tetracycline.[1-4,5-7] They are responsible for causing mutation, antibiotic resistance and pathogenesis. Radioisotope labelled IS1, IS2 and IS3 DNA sequence probes with $0.8 \mathrm{~kb}$ to $1.2 \mathrm{~kb}$, DNA sequence could support the process quickly and accurately. Bacteria, Fungi, Mould and Algae are belonging to organised (eukaryotic) nucleic acid cells. Our body cell system belongs to eukaryotic cells and tissues. Viral cells are different. Viral cells as prokaryon, unorganized (prokaryotic), carry RNA and DNA ss, (single stranded), or ds (double stranded) coated by protein. They are nano and micron level big and are air-, dust-, insect-vector-and water- born. Their functionality will depend on host, their survivals depend on host. Based on host specificity, they can be living and pathogen; otherwise they get abolished in the long run. For survivals virus change their functions by mutations to sustain in evolution. All prokaryotic and virus have partial and full parasitic nature. It proves that DNA could have enormous power to adapt the environment. In support of bacteriophages (like Lambda, Psi-ex, T4 viruses) specific to bacteria, they maintain their existence in different animal viruses. Like. Adenoid-, retro- and rhinoviruses, specific to human, animal, birds and insect for host specific growth and transmission, $[\mathbf{5 , 6 ]}$. Virus cannot survive long, in scientific point of view, since they have no cell wall, only by protein and lipid coat, they remain dormant inactive. By epigenetic manipulation viruses manipulate single (ss) and double stranded (ds) DNARNAProtein propagations in host specific animal cells. As per the laws of Darwin and in the thrust of survivals, they aggressively search host, as non-infective, long and short term infections, [7-10]. Spanish flue, Influenza, MARS, SARS,HIV are those examples.IS1, IS2 and IS3, or other Tn Sequence, if found, could be used as DNA short sequence probe and could be used to develop antibodies, specific to corona MERS, SARS, HIV viruses. Methods of probe-DNA isolation have been discussed, [1-5, 7-10]. Although the mode of infection and transmission of these viruses are different, namely, air, dust,-water, human and animal born, mutations have accomplished them to adapt and to search matrix/ vector as carrier (i.e. mosquito born Dengue) in Asia/India migrated from USA, Latino America and South East Asia, different than corona. - The most important part of these viruses is the coat (protein or lipid) and their epigenetic function of RNA and DNA, supported by ss, ds, replication, transcription and translations. The mode of Infectivity, rapidity, and sustainability of these viruses are basically based on such coats and by their adaptive mutations of the host cells, their propagations, long and short distance. Viruses are cleverer, compared to bacteria. For designing vaccine against such infective agents, IS mediated hybridization could be essential to classify them in adeno (DNA) and retro (RNA) viruses, including corona. By cloning HA gene of corona intoE. coli , the surface antigen of hybrid E.colifimbriae/pili could be used for humoral memory based response of Tand B-cells and as cytotoxic Tc and Tk cells. Since IS could be available both in mutations and in wild types, [1-7], evolution of virus is supposed to be caused by the fragments of nucleic acids of the host cells, Fig.1-5]. 

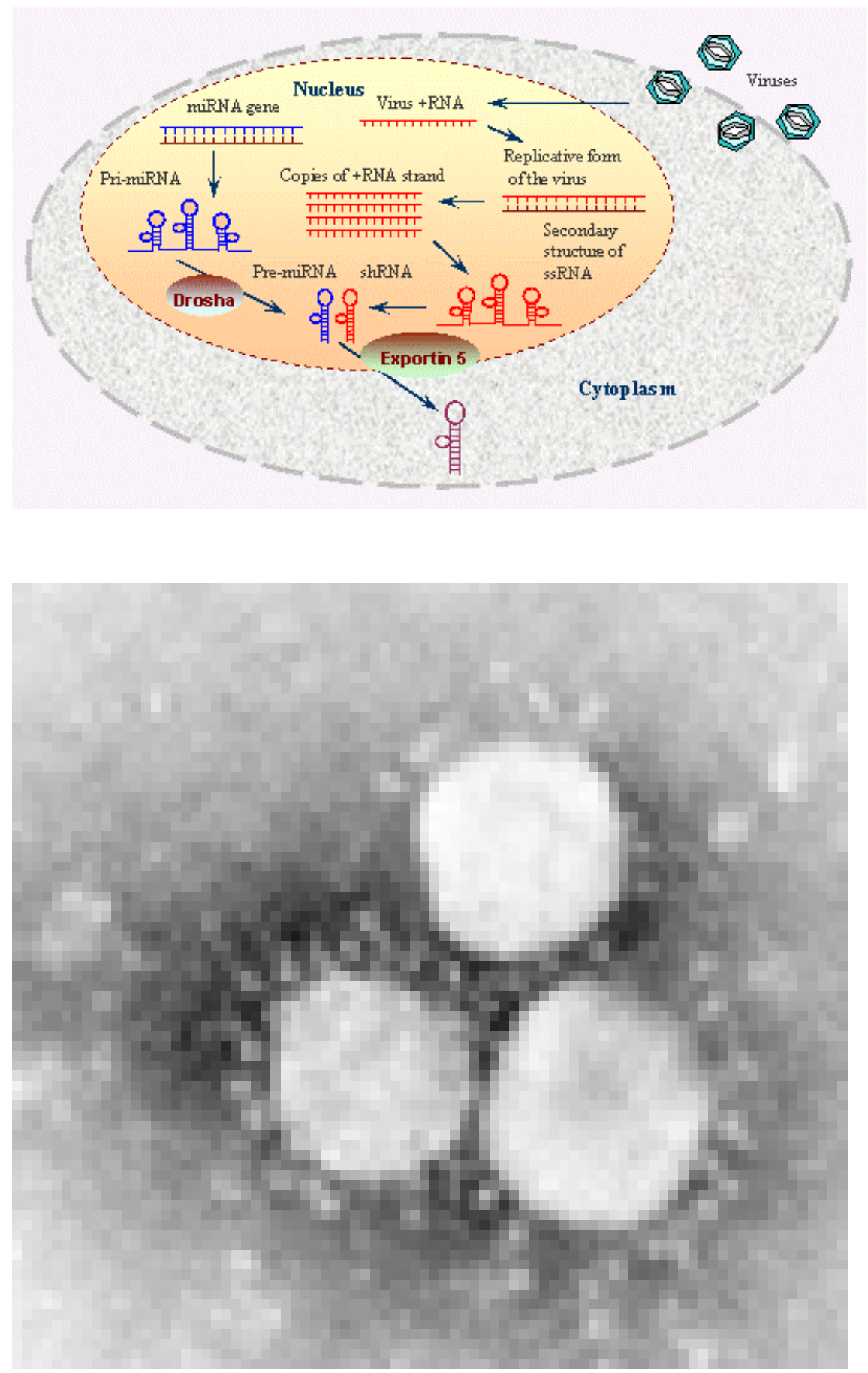


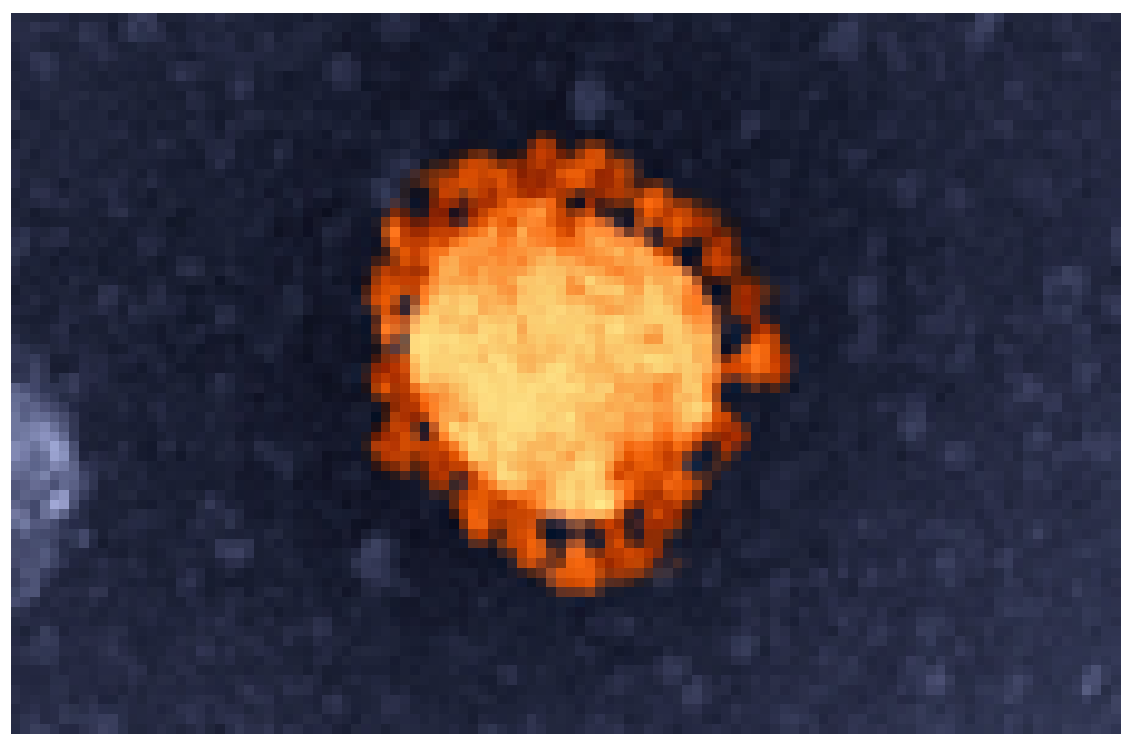

\section{Inrikgedytur}

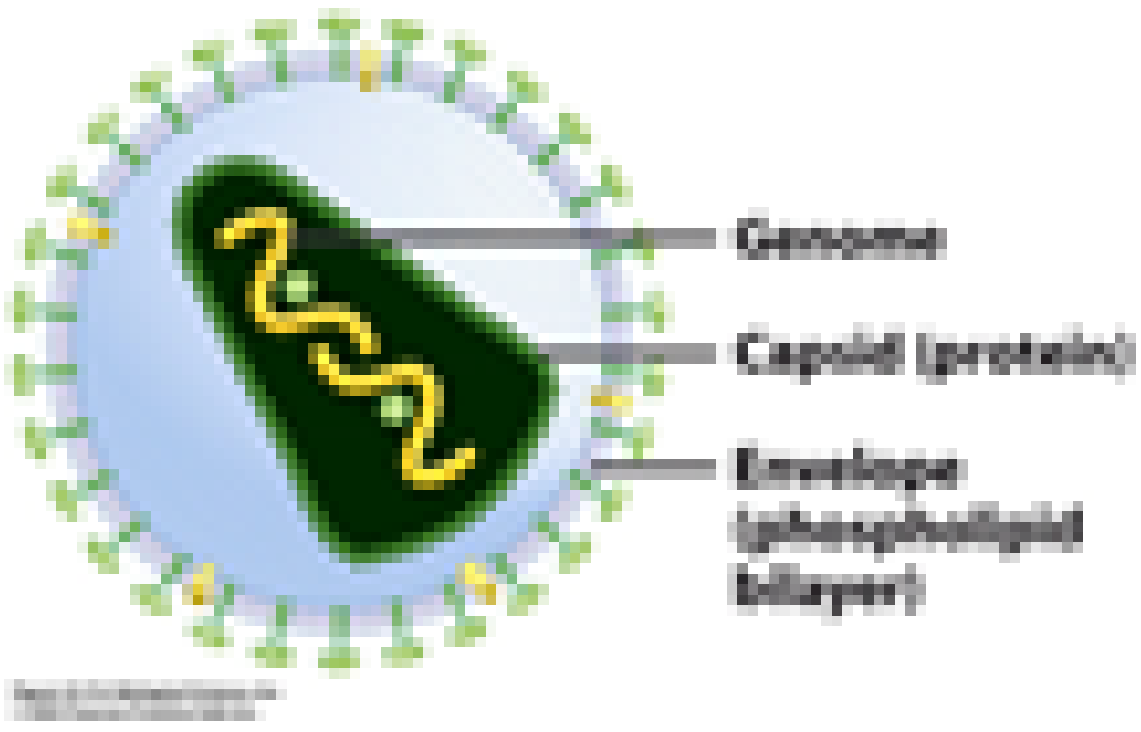




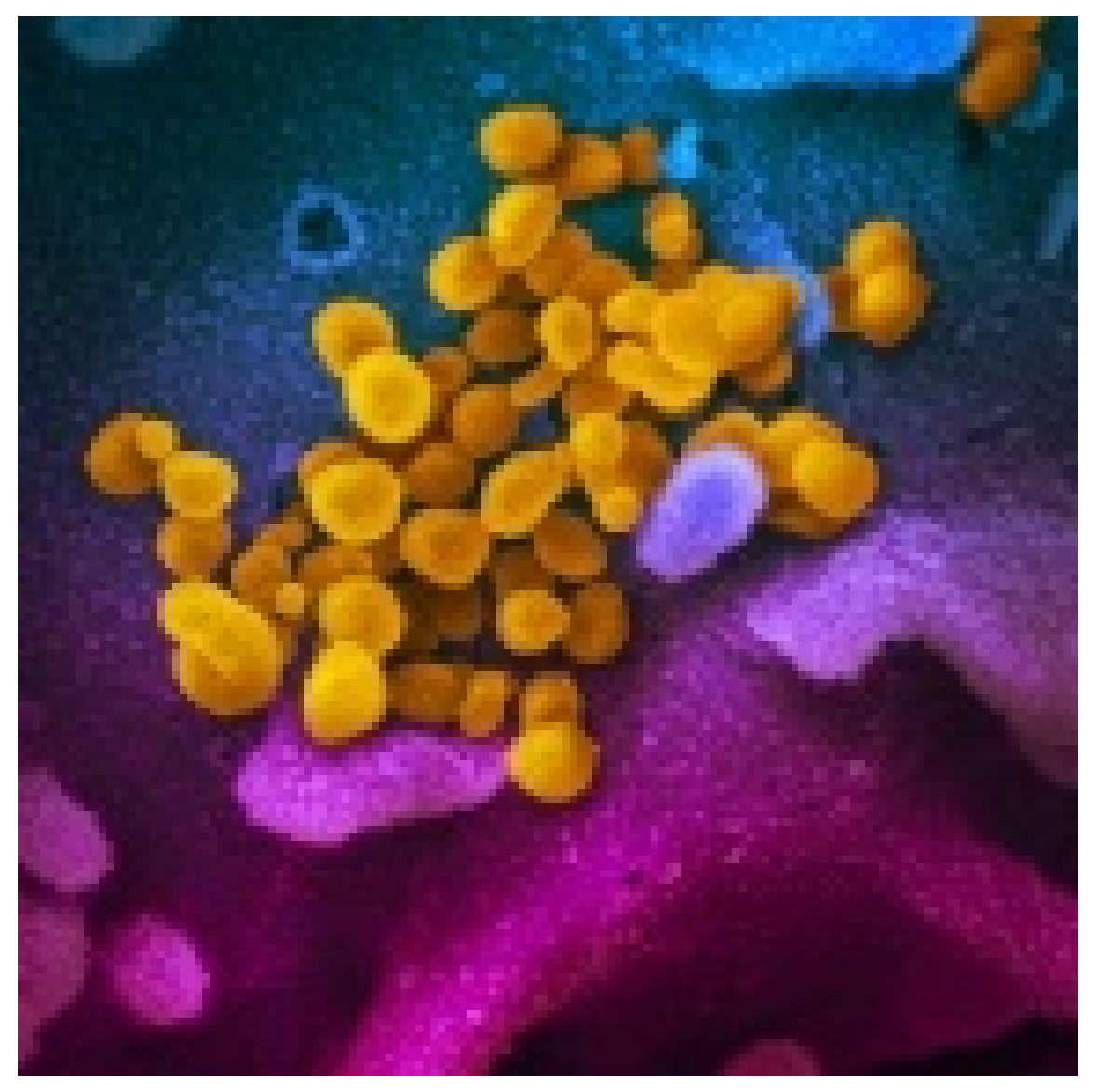

Fig.1. Fig.2 Fig.3 Fig.4 Fig.5

Fig.1 describe, the possible mechanism the formation of virus particles through NA( Nucleic Acid) fragments, Fig.2, and 3, SEM view of Corona, and H1N1 virus, Fig. 4 shows the molecular structure of SARS-COV-2 RNA-virus, Fig.5 shows the SEM view of Wuhan corona clustered .

SEM derived images of Covid-19, SARS-Cov-2, and H1N1 virus shows the similarities of surface antigen, spike protein, where Wuhan corona show no spikes. The spontaneous reaction of viruses, specially corona can be speculated, that due to ss RNA, it will immediately transcribed to form capsid protein and for the multiplications of virus particles in lung cells, using r-RNA of the infected lung cells, after penetration, virus uses 3' end of ss mRNA and floating r-RNA of the host lung cells to generate envelope. Envelop of Corona is made by glycoprotein as matrix protein. It encapsulates ss RNA of the replicating ssRNA of the virus, after fusions of coat- glycoprotein with lung cell surface membrane attachments OC43 haemagglutinin type virions are released from host cell, as reported, ruptured cell membrane. New virions of corona, transmit the infection via airborne droplets to the healthy recipients. Virus replicates/multiply locally in cells of the ciliated epithelium, causing cell damage and inflammation. The appearance of antibody in serum and nasal secretions follow the infection and increase immunity peaks in the winter as local epidemics and could last for a few weeks or months. The same serotype may return to the same locality after several years. The virus is difficult to isolate. Nucleic acid hybridization tests (including PCR) are now being introduced as control treatment of common colds and within a-symptomatic patients. No vaccines or specific drugs are available till date. Only vaccine with IS element approach could be an alternative solution to face the present crisis, if at all be successful, [7-10]. Coronaviruses are found in avian and mammalian species. They resemble same 
each other in morphology and in chemical structure. Even coronaviruses of humans and cattle are found same in their antigens, there is no evidence till date that human coronaviruses can be transmitted from cattle and from animals. In animals, coronaviruses invade many different tissues and cause a variety of diseases, as respiratory infections, i.e. common colds. On rare occasions, gastrointestinal coronavirus infection has been recorded among children, but these enteric viruses are not well defined. After an incubation period of about 3 days, corona virus can cause the symptoms of a common cold, including nasal obstruction, sneezing, runny nose, and secretions. Presence of corona in multiple sclerosis are also not well defined including their infections, [11-17]. Wuhan corona SEM picture, Fig.5, represents the similar 026: EPEC bacterial cluster, as they were reproduced by the author during 1983. The curiosity remains, that whether they have the similar adhering and invasive, colonizing nature, as corona on lung epithelial cells, as in 026: EPEC, inspired the author to recapitulate, theoretically the colonial invasive nature of corona as in 026: EPEC. The question is whether, corona has procured similar gene (haemagglutine) of adhering and invasions as in 026: EPEC.

\section{Corona vaccine proposals and their development:}

As the Wuhan corona virus (COVID-19) epidemic continues to spread and there is still no solution, their preventions (i.e. any vaccine, any $\mathrm{T}$ cell based cytotoxic activities on corona. At present there are many pharmaceutical companies and government agencies are working hard to resolve the problem; one strategy to use a live but weakened (attenuated) virus, as found in chickenpox vaccines. The second is to use a dead (inactivated) virus, as found in flu and polio viruses. The third one is to use only a piece (subunit) of the virus, as recovered from HPV(Human Papilloma Virus) and shingles, a viral infection caused by varicellazoster virus, painful rash with blisters., vaccines. The first two methods require growing the virus in a laboratory or factory. This isn't as easy as culturing bacteria, which usually grow just fine if provided the right types of food. Viruses can't live on their own and must infect another cell in order to reproduce. So, to culture viruses, scientists must first identify a cell type that can propagate them. Then, along with their host cells, the viruses can be grown inside large tanks (bioreactors). Alternatively, many (possibly including the new coronavirus) can be grown inside eggs. For live-attenuated vaccines, viruses were inactivated by heat or by chemical like formaldehyde. Sino Biological, Chinese biotech firm, explains that growing corona viruses poses a safety threat to lab workers. The danger in such vaccination is, that if the virus is not properly attenuated or inactivated, it could provide reverse effect. This has happened with polio in Africa. Report says that subunit vaccines would be safest, since they do not carry any protein unit of virus coat of whole viruses. Subunit vaccine needs bolstering system and proper knowledge of virus, (i.e. the types of virus, their DNA and RNA structures, ds, or ss, their genomic sequence, and their mutational variations, as isolated within infective and non-infective phases). Once this is accursed, then to use same virus in engineering and in attenuating protein vaccines will not be problem. The French pharmaceutical Giant Sanofi is using this method to target COVID-19.In addition to that there are newer methods to design vaccines, and several companies are testing them in the race against COVID-19. Johnson \& Johnson is using genetic engineering to modify a harmless adenovirus to resemble COVID-19. Inovio is developing a DNA vaccine, while Moderna is creating an mRNA vaccine. (This diagram depicts how DNA and RNA vaccines work), Fig.6 (a). Fig.6 (b), represents the procedures which could be considered. The steps are made in following manner;

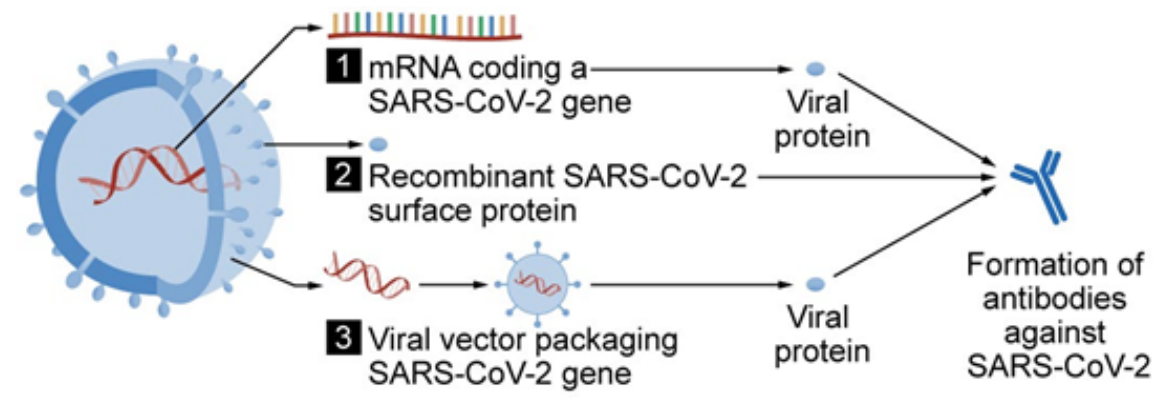

Source: GAO. | GAO-20-583SP 


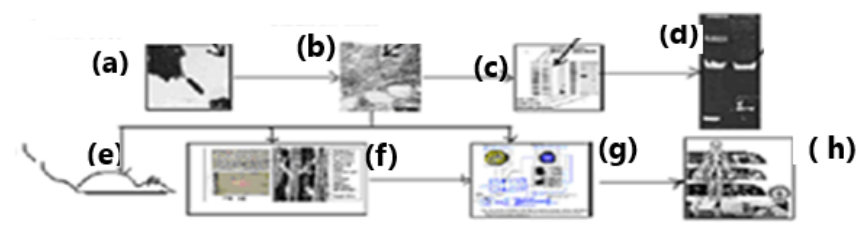

\begin{abstract}
A complete step, starting from (a) SEM view of 026:EPEC,(b) their isolated view of fimbriae(pili) in SEM,(c)their protein, (d) their plasmids(e)the mice experiment both by $(f)$ isolated fimbriae and $(g)$ viable hybrid genetically engineered cells, and finally (h)the proposed human application.
\end{abstract}

Fig.6(a ) (b)

Fig.6 (a)-b) A combined afford will be taken to establish the concept. The author personal work on AAIR in Balb/ c mice is considered, with the objective that genetically engineered plasmid with spike protein gene and IS-Tn-IS may be used to target the corona virus specifically, where the spike protein gene will signify, the existence of corona, and IS-Tn-IS will signify, the presence of IS element in the infective corona, mutated and are varied and are escaping from the body humeral immune system ( i.e T-B-Cells and Macrophages and their associated, Interleukin(IL1-IL-10, Interferon (IF), killer( Tk, NK) and cytotoxic (Tc) Memory( $\mathrm{Tm}, \mathrm{Bm}$ ) cells for sustaining the recognizing the mutations and the same could be identified by the existence of any IS-Tn-IS sequence ( IS1,IS2,IS3 . . IS10).

\title{
Classification and Antigenic Type:
}

The corona viruses were originally grouped into the family Corona, is known as crown constituted by glycoprotein-studded envelope,Fig.2-4. Most human corona virus fall into one of two groups of $229 \mathrm{E}$ and OC43-like. These differ in both antigenic determinants and culturing requirements: 229E-like corona viruses can usually be isolated in human embryonic fibroblast cultures; OC43-like viruses can be isolated from suckling mouse brain. There is little antigenic cross-reaction between these two types found, and cause independent epidemics. It is thought that human coronaviruses enter cells, predominantly, by specific receptors like amino peptidase- $\mathrm{N}$ and sialic acid-containing receptors.229E and OC43 respectively antigenic variants during infection enter the host cell and release their genome RNA to transcribe and to translate. (Messenger) mRNAs of the host lung cells and their 3' ends. 5' ends are used for translating coat protein. There are 7 mRNAs are involved in such envelop coat proteins development and are assembled at the cell membrane. The genomic RNA is involved to develop virus particles and to get released by budding from internal cell membrane ruptures. Studies in both organ cultures and human cells, it is observed that infected cells become vacuolated. Cell damage triggers the production of inflammatory mediators, which increase nasal secretion, local inflammation and swelling to stimulate sneezing, obstruct the airway, and raise the temperature. The growth of several opportunistic, Bacillaceae, Coccaceae, Chlamydomonas, of microbial family, influence the increasing inflammation, symbiotically survives with corona to damage mucocilliary activity of the respiratory tract and lung, and to bring the patients to death,[18-19]. Interferon(IF) can protect against infective stage, but its expression in human immune system with MHC( Major Histocompatibility Complex- HLA ( Human Link Antigen) and IL( Interleukin10(IL10), is not well defined in case of corona. Because coronavirus infections are common, many individuals have specific antibodies in their nasal secretions, and these antibodies can protect against infection. Most of these antibodies are directed against the surface projections and neutralize the infectivity of the virus. Cell-mediated immunity and allergy have not been studied vast to confirm the mode of corona allergic inflammations. The epidemiology of coronavirus colds is not well defined and studied. Hard Immunity concept is an alternative infection movement, pass through communities, and occurs during winter months in families, schools, and locality to block corona infection. [11-15].

Vaccine Model interpretation and experimental proposals. 
Volunteer's experiments show that coronaviruses are extremely fastidious and grow only in differentiated respiratory epithelial cells.More than 90-100 vaccines are being developed against SARS-CoV-2 by research teams in companies and universities globally. Researchers are using experiments and begun injecting formulations into volunteers in safety trials; others have started testing in animals. Fig.7,describes the statistical distribution of various virus vaccine.Fig.8, describes the hybridization in plasmid and chromosomes by the author. IS1 DNA, cloned in vector plasmid to search AAIR in Balb/c mice. Bottom figure shows hybridization effects reproduced inEscherichia coli $k$-12 C600 Yale strain hybrid plasmid, chopped by restriction enzymes. Fig.9 describes the procedures of very accurate identification of gene presence in hybrid gene by Southern hybridization

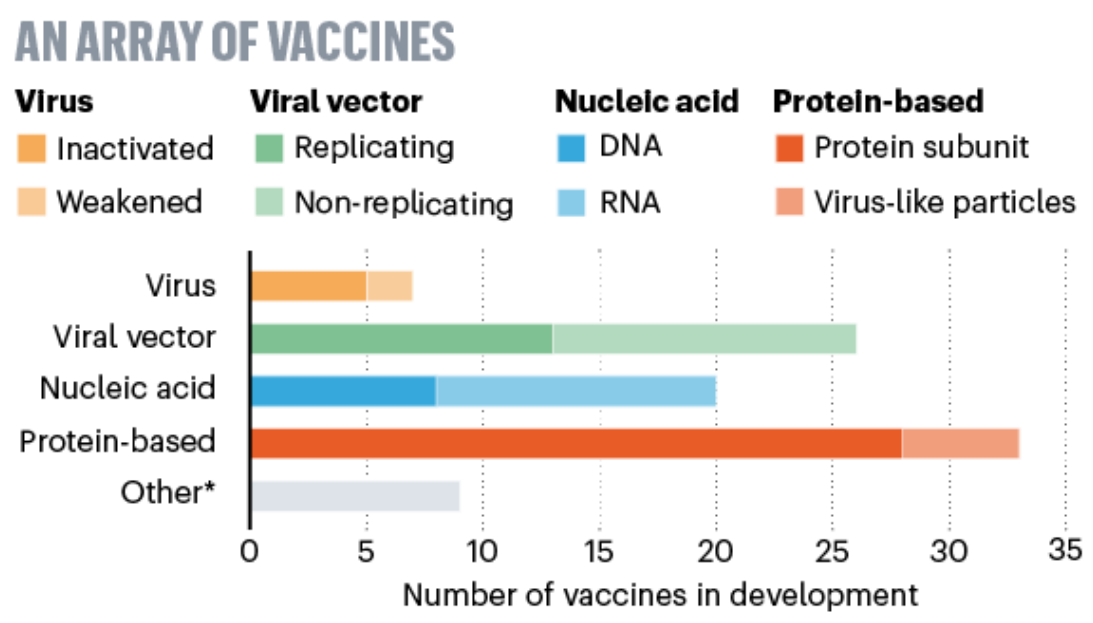

* Other efforts include testing whether existing vaccines against poliovirus or tuberculosis could help to fight SARS-CoV-2 by eliciting a general immune response (rather than specific adaptive immunity), or whether certain immune cells could be genetically modified to target the virus. 

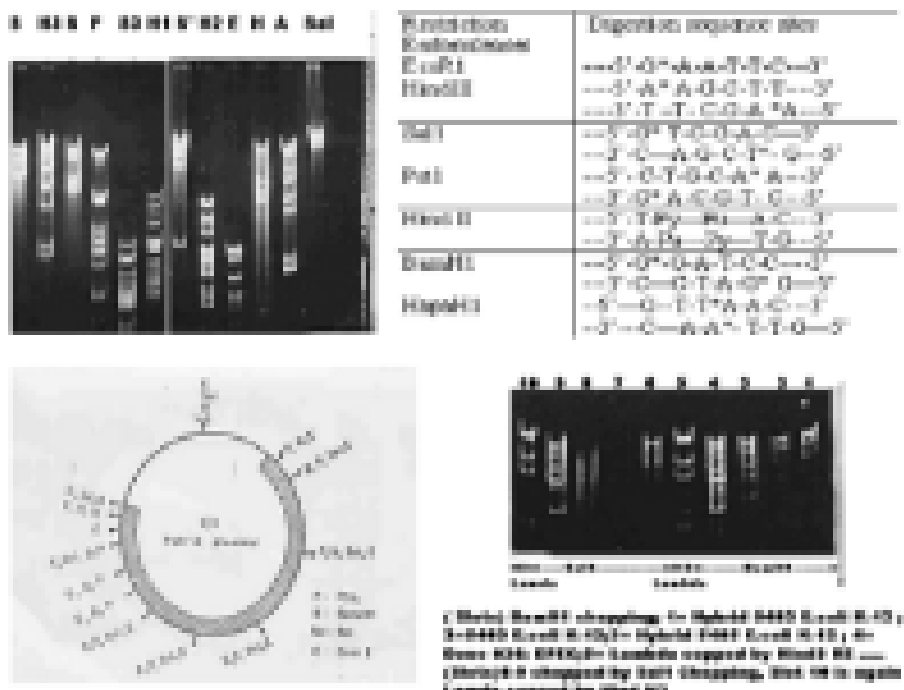

Bripga intiti marr that

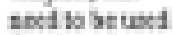
furitrpe phe hter Fhadine 미 Hat सम대 4. mithingm furty deprista hrmin

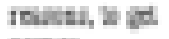
새를

frmati [HHW

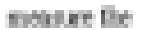
ind inds pribute promen of PMHdB: mirlnatir

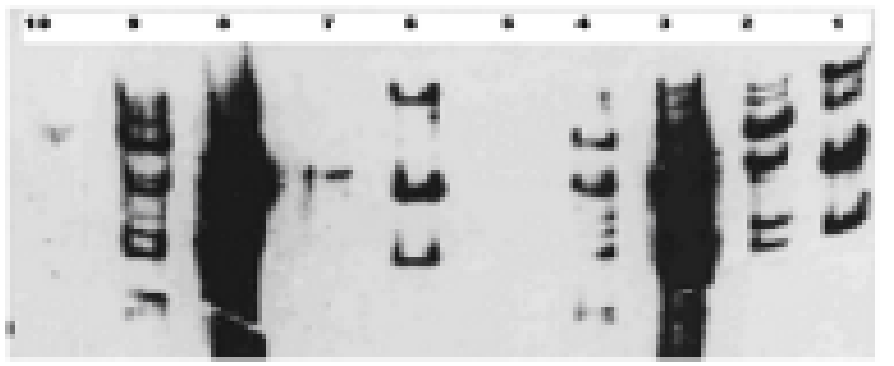
aretp. ISI. Leth atrond phatar marst un

7Th=nh AHT int Dethr dowt mirlyst

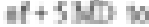

MTPT

Silified

\section{Southern blot}

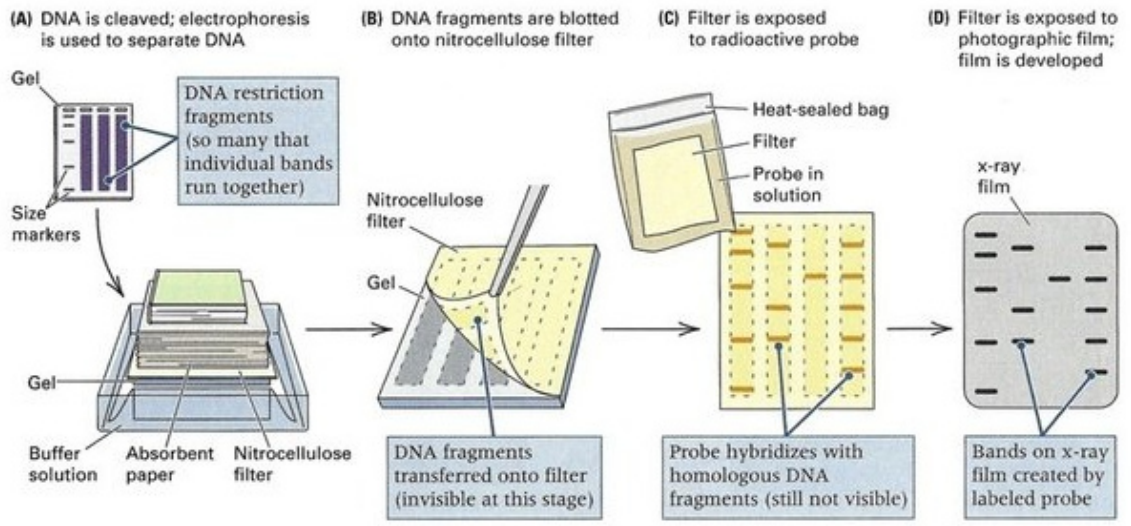

\section{Fig.7 Fig.8 Fig.9.}

IS mediated viral spike gene cloning has been proposed and reproduced in following manner by the author. IS probe was isolated purified from the known source of E.coli $k$-12 . The same probe could be used to search the presence of IS in corona, SARS, MERS, HIV, Influenza viruses to short them in groups. IS(Insertion 
Sequence) DNA probes were p32 alfa d ATP labelled as ss stranded DNA, and were hybridized against ss DNA, and ss RNA of viruses. Southern and Northern blotting both could be used as per the need, Fig.9. Prof. Barbara Mc Clintok of USA, discovered Tn in Maize seeds by their segregated color changes and named Tn ( Transposable element) Later Prof. P.Starlinger studied the same in Bacteria E.coli, Prof. H. Saedler as his follower continued the same. The integration and multiplications/ jumping from one place to other, from one gene/ plasmid to other could be initiated by IS elements and their repeated and inverted repeat sequences. The searching of IS element in corona could similarly be made as proposed. DNA of hybrid strains would be studied separately. By isolating their chromosome DNA and Plasmids, the presence of copies of IS element was studied. 8 copies of IS1 and number of copies of other IS elements were varried including the sequence lengths. The changing the number of IS copies in hybrid chromosome and plasmid will confirm, the cloning of viral spike gene by copies of IS elemens, if compared with IS copies of the original recipient E.coli $k$-12 C600 Yale strain, already existing with 8 copies of IS1 in E.coli chromosome.. In case of hybrid these copies will remain same or will change, Fig.10.

\section{Insertion sequence}
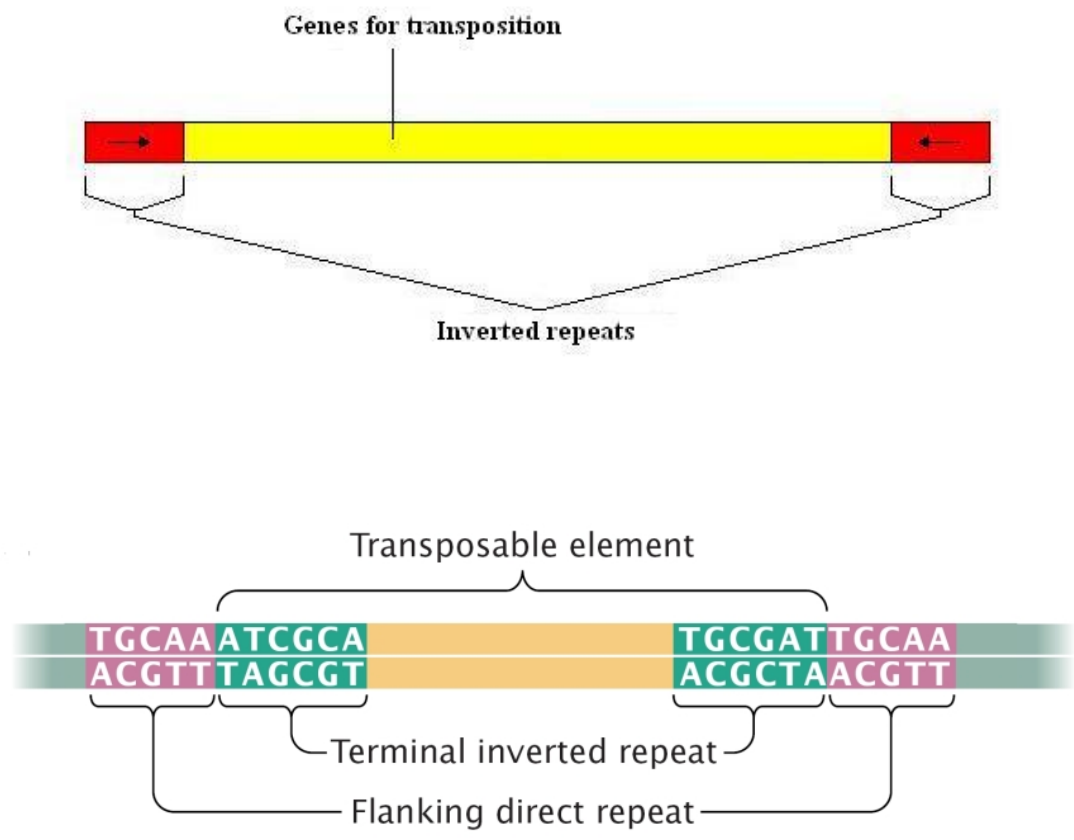

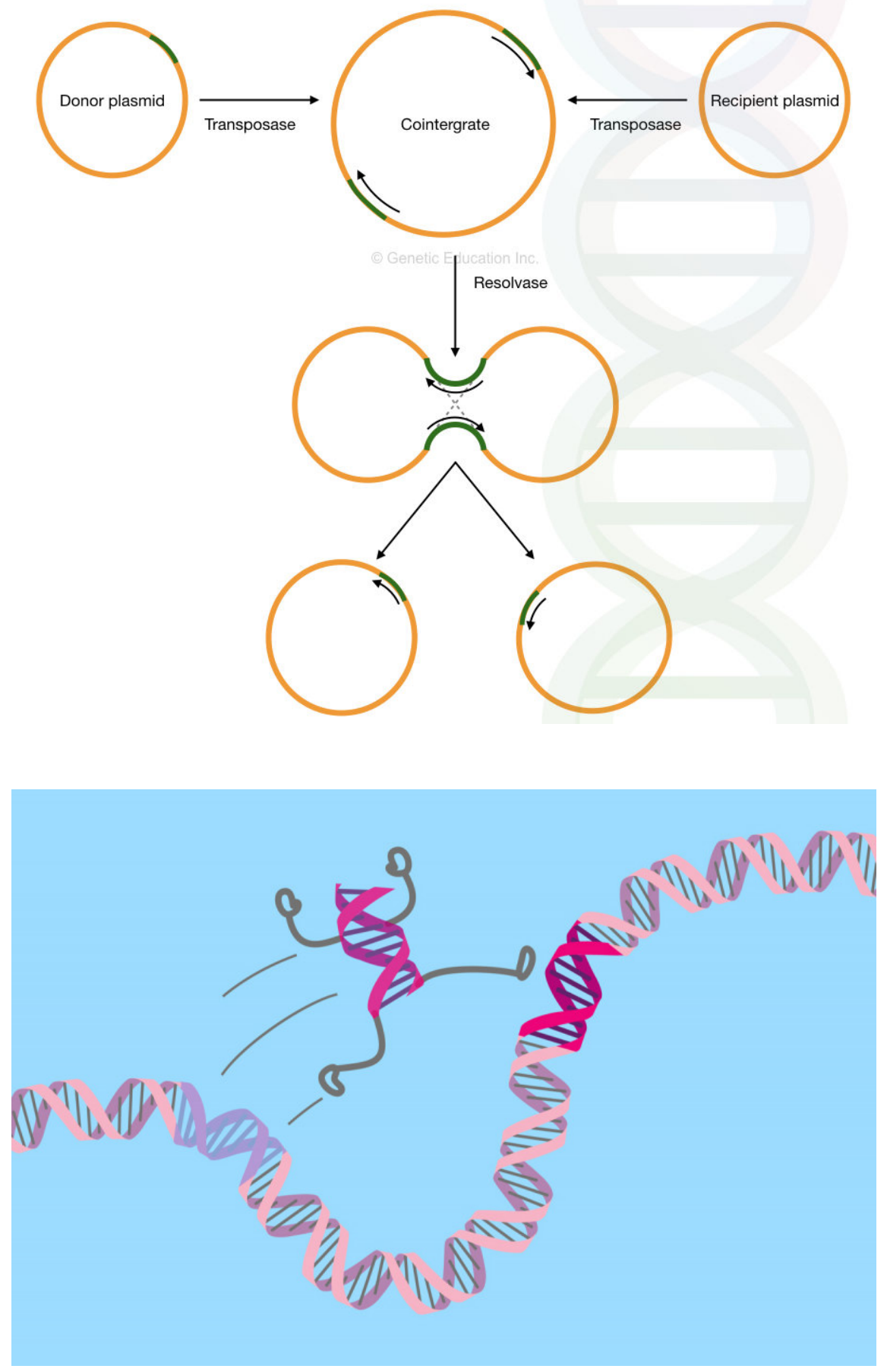


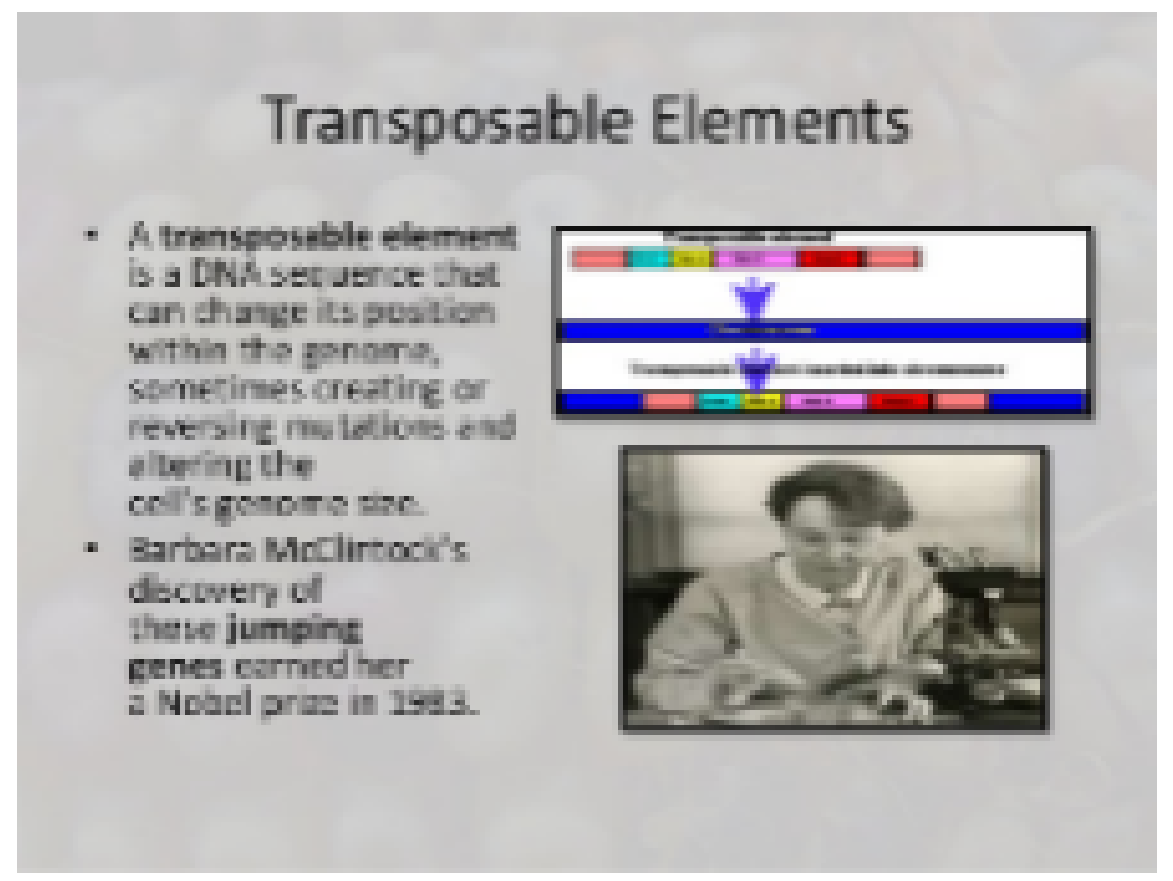

Fig.10(a) (b) (c) (d) (e)

Jumping genes, Fig.10(a-e), [ 1, 4-7,8,9-16], (a) shows the structure of one Tn, flanked by IS elements Left and Right end to Tn palindrome, (b) repeats are identified by the sequences of IS elements, repeat and inverted repeats of IS (c) Recombination and sequence multiplications of IS-Tn-IS is reproduced in plasmid and chromosome hybrid combination (d) Animated concept of jumping sequence of DNS sequence, (e) Historical discovery of Tn by Barbara Mc Clintock.

IS based Tn (Transposons) are involved in viral evolution, mutation, camouflaging infective nature of SARS COV-2 virus, corona[9-16]. Jumping-genes as retro transposons [8]hijacks special cells called nurse cells, produce invasive nature of DNA driving evolution, and causing disease. Almost half of our DNA sequences are made up of jumping genes, known as transposons. They jump around the genome in developing sperm and egg cells and are important in cellular evolution [4-7] and cause new mutations that lead to diseases. Remarkably little is known about when and where their movements occur in developing reproductive cells, the key process that ensures their propagation in future generations but can lead to genetic disorders for the hosts. Animals have developed a powerful system to suppress jumping gene activity that uses small, non-coding RNAs called pi RNAs (Piwi-interacting RNA (pi RNA) is the largest class of small non-coding RNA molecules expressed in animal cells, through piwi -subfamily Argonaute proteins. which recognize jumping genes and suppress their activity. Occasionally, jumping genes could mobilize virus infection, propagated from Wuhan, China, to all over the world without any variations of RNA. Measurement of antibodies to SARS-CoV-2 will improve disease management if used correctly. In late 2019, China reported a cluster of atypical pneumonia cases. The causative agent was identified as a new beta coronavirus, called severe acute respiratory syndrome. The virus rapidly spread across the globe and caused a pandemic. Sequencing and IS hybridization of the viral genome allows the development of nucleic acid-in hybrid and wild types, that could have been widely used for the diagnosis of acute (current) SARS-CoV-2 infections [19].

\section{Conclusion;}




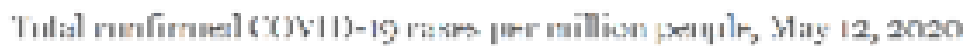

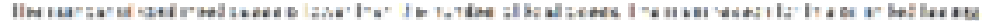

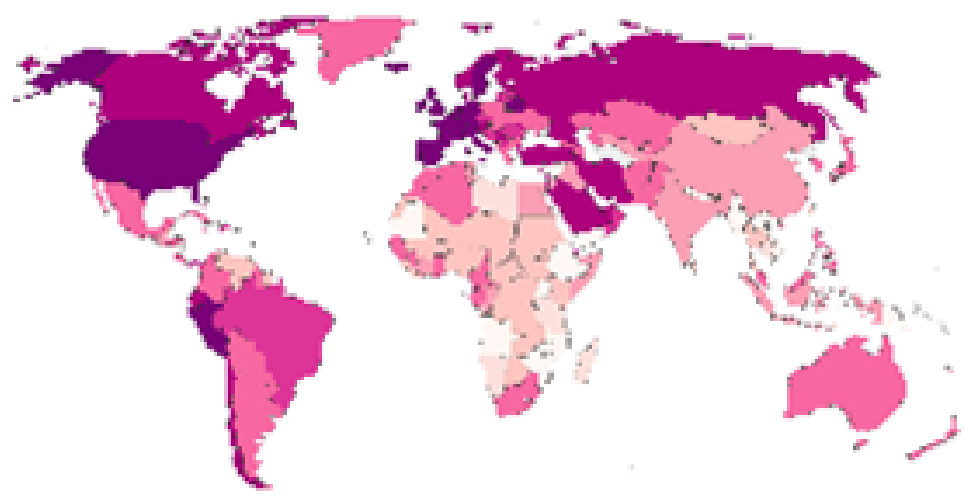

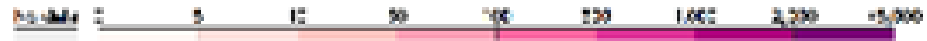

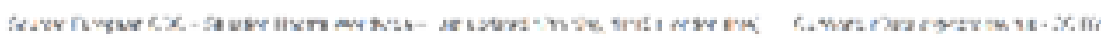

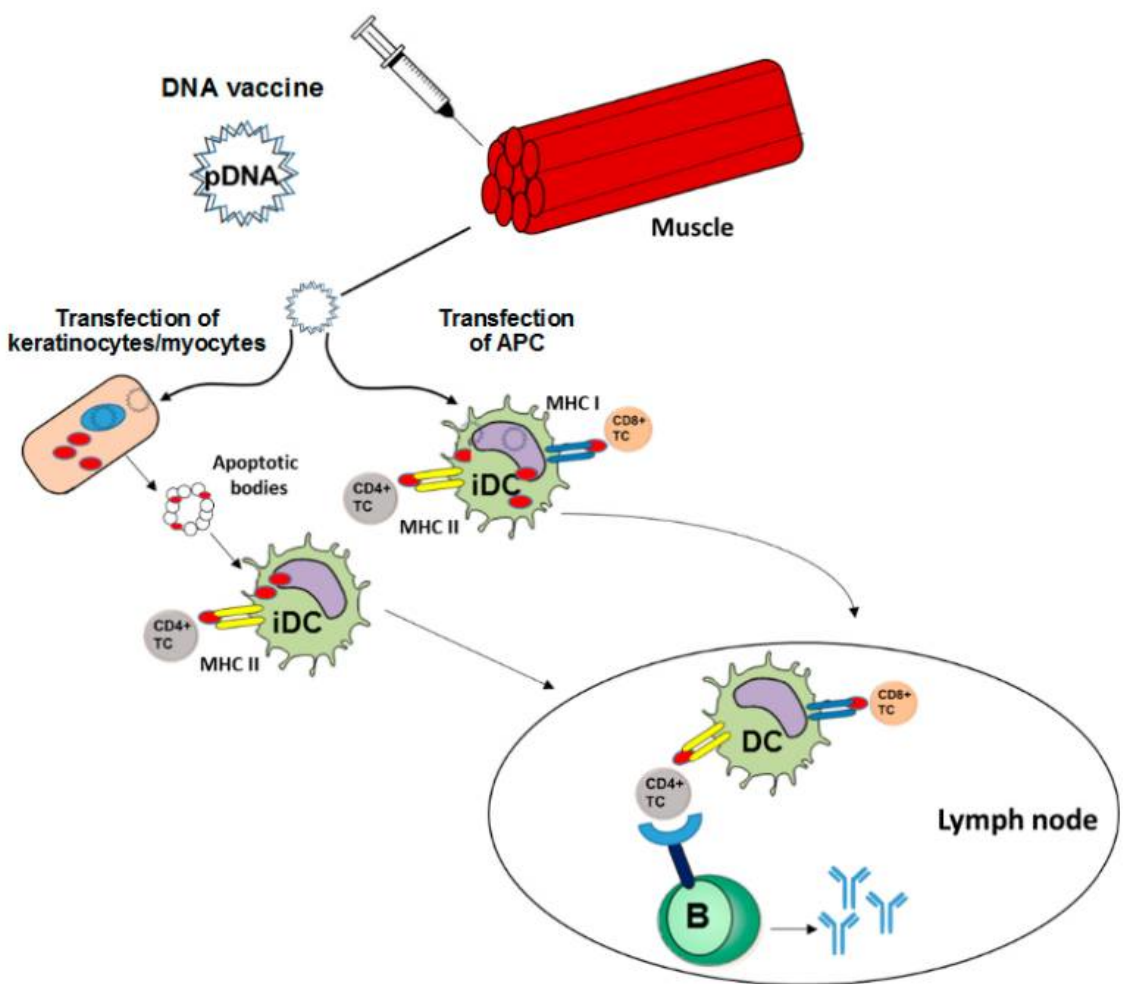




\section{Potential pandemic size and herd immunity The fraction of the population that becomes infected with a transmissible disease in a simple epidemic model increases nonlinearly with the intrinsic reproductive number, $R_{\sigma}$ and will exceed the threshold for herd immunity. $R_{0}$ is the expected number of cases caused by an index case. Interventions can reduce $R_{0}$, the total fraction of the population infected, and the threshold for herd immunity.}

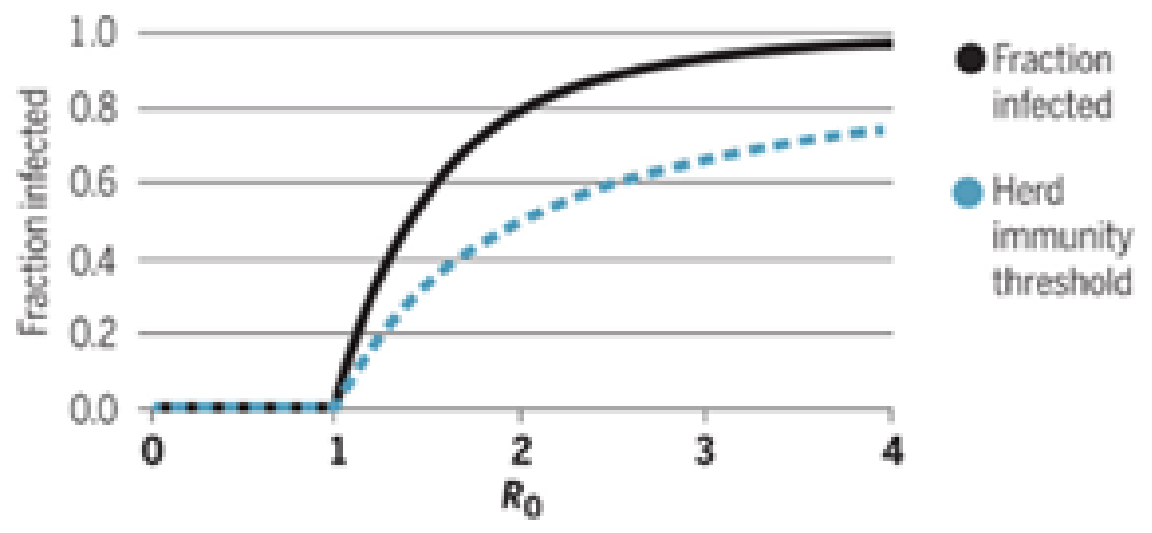

Fig11(a-c) ) (b)(c)

Graphic adapted by N carry /Science, Fig .11(a-c), describe the data for total confirmed COVOID-19 patients globally. Pink VioletDark Violet, Dark -Violate represents the most affected area of USA, Europe.Fig 11(b) shows the future perspective of DNA Vaccine, injecting to muscle and their immunological response to MHC, CD8, CF4 and B-Cell.Fig.11(c) represents, the conceptual propagation of "Herd-Immunity". Herd immunity is still remain as speculation since the rapid mutation of corona. DNA vaccine model would also be difficult, since the mobility of corona is spontaneous. Based on the above information the author has taken some initiative to discuss the present crisis of corona, would be different and where the spontaneous mutations are occurring. The mischievous/ unknown spontaneous killing nature of SARS COV- 2 virus, n-COVID-19 infections can only be prevented by vaccinations, where monoclonality in humoral and cytotoxicity of antibodies are concerned and should be spontaneous, and memory based. One proposal IS mediated antibody development could be feasible as proposed by the author It is to be mentioned that humoral and cytotoxic natures of vaccinations are concerned. Since Corona is pandemic. Humoral types would be used for the future generation and cytotoxic types would be used for present situation, for those, who are infected and are being separated as a-symptomatic, co-morbid class., the Corona pandemic or endemic infections cover many and vast knowledge of information and understanding. Starting from medical and biological sciences, corona treatment needs also good understand of physics and engineering, the movements of molecules and fluids in and around respiratory system. The concept of microfluidics, the molecular transport, the concept of pressure and temperature driven molecular transport, all would be considered to consider the design concept of cytotoxic vaccine of corona. In lung, alveoli, where the adherence of virus is predominant, the adherence mechanisms of corona spikes and its protection by humoral antibodies would perhaps be the ultimate vaccinations of corona. On the surface of lung cell spike proteins of corona manage the biochemical functions of ACE_I and II in cell receptors of lung cell damage. It is also not possible to design vaccine, when virus is already in entry, because virus corona expresses many antigenic variations inside the body. The design of vaccine represents the molecule responsible for humoral immune response must be well known. However, the author believes, that AAIR (Anti-adherent Immune Response) against corona could one of 
the solutions. Cytotoxic immunity does not allow to prevent the entry of corona virus inside the lung cell and or any organ specific epithelial cells it will only prevent the growth of corona. Humoral antibody in the human body as AAIR would be appropriate at present to design vaccine with IS sequence to prevent corona infection. $[\mathbf{1}, \mathbf{4 - 7}, \mathbf{1 0 -}, \mathbf{2 0}]$. Till date no vaccine has been developed [21-25 ]. Corona was known to the scientists and medical practitioner since 1978[10], page 619, but its epidemic and pandemic nature were not discussed. So it might be considered to realize, that corona similarsers-cov- 2 virus is continuously mutated towards. MERS, influenza, H1N1, Swine or Chicken flues and returned to returned back to present corona stage and in between transformed to insect vector-borne diseases, like mosquito as Dengue and Ebola by some other vectors.IS-Tn-IS might have some reason to search and to establish polio like oral vaccine, ultimately to win from all such mischievous, viral culprits and to save the mankind. So long the mechanism of infection of corona is not properly understood, it would be difficult to design corona vaccine. The basic objective of the scientists would be to protect the entry of corona virus, i.e. to design AAIR through IS mediated DNA RNA manipulation of corona virus would be one way.

\section{How Close Is a Coronavirus Vaccine?}

Engineered virus might be able to block coronavirus infections. Mouse study shows many responses. A new intranasal vaccine using an RNA virus for gene delivery protects against fatal MERS coronavirus infections in mice. The mice were genetically engineered .All the mice that received the vaccine survived MERS, while the mice that had not received the vaccine did not. The researchers are now applying the same strategy to develop a vaccine for SARS-CoV-2, the virus that causes COVID-19, which has infected more than a million people worldwide, signified the challenge for finding an effective vaccine against the coronavirus that causes COVID-19, left only the race against time, McCray said once, that, one hundred percent of the population if not exposed by the virus, that does not mean that there will be no people or more people will not be infected when it comes again,". It is very difficult to say against lasting immunity against SARS-CoV-2 infection, so it's important to think about vaccine to protect the population. "The American Society for Microbiology is one of the largest professional societies, dedicated to microbiology, life sciences and is composed of 30,000 scientists and health practitioners. [22 ], emphasize also for corona-vaccine. Several pharmaceutical industries are recently involved to develop corona vaccine. "Moderna" set a drug industry with mRNA-1273, a vaccine candidate identified recently after the novel coronavirus was sequenced. "National Institutes of Health "USA describes mRNA-1273 could be used to develop monoclonal antibody and the same could be used safe to prevent corona. "Moderna" took the responsibility to use mRNA. "Food and Drug Administration", USA approved the use of mRNA medicines. "Germany's Biotech" is working on an mRNA vaccine for the novel coronavirus, mRNA to spur the production of protective antibodies. "Shanghai's Fosun Pharma" jointly moving with Biotech's vaccine in China. "Pfizer" participated in the program to co-develop the vaccine in the rest of the world. "Takeda" is approaching preclinical treatment, using blood of people who have already been infected by the coronavirus. Spanish Flu pandemic of 1918, "Takeda" emphasizes, that plasma treatment could take responsible phase in treatment corona patients. According to the company, the therapy could be available to patients in 12 to 18 months. "Johnson \& Johnson", applied vaccines against Ebola and Zika virus. The company is in the early days of developing a vaccine against corona. Human trials could begin by November. At the same time, J\&J is working with the federal Biomedical Advanced Research and development authority on potential treatments for patients who are already infected, a process that includes investigating whether any of its older medicines might work against the corona virus. "Glaxo-Smith-Kline', one of the world's largest vaccine manufacturers, is lending

its technology to a chinese biotech firm at work on a coronavirus vaccine. Stanford University, Schools of Engineering \& MedicineBottom of Form

, approaching and took the initiative on CRISPR(clustered regularly interspaced short palindromic repeats).CAS(CRISPR associated protein)-9 technology.Crisper-cas-9 will work as transposons and will detect the presence of coronavirus, protein to repaire.The Stanley Qi Lab is exploring ways that Crispr technology can be used to fight the coronavirus pandemic. Their latest results are one piece of a larger puzzle. March 18, 2020.Stanley Qi, a pioneer in Crispr technology tools. However, one challenge is still remain, how long we have to wait, and whether: "Could Crispr Be Humanity's Next Virus Killer?" , the" Development 
of CRISPR as a prophylactic strategy to combat Coronavirus and Influenza. Different approaches have been discussed, [25], on s pike protein by CRISPR-CAS-9, RNA based spike protein modifications. Scientists are developing hundreds of coronavirus vaccines using a range of techniques, some of which are well-established and some of which have never been approved for medical use before. Whole-Virus vaccines inactivated and live attenuated vaccines, genetic vaccines are being partially used against coronavirus, using genetic code of virus and human hosts. RNA vaccines with viral vector vaccines. Vaccines using adenovirus or other viruses protein-based vaccines, vaccines that use against coronavirus. Protein or a protein fragment, recombinant vaccines, yeast or other cells can be engineered to carry a virus gene and to generate viral proteins, which are then harvested and put into a vaccine, a coronavirus vaccine of this design would contain whole spike proteins or small pieces of the protein. This category includes some vaccines for shingles and hepatitis B. However the author's IS-Tn-Tn recombined by spike protein cloned plasmid, transformed into Escherichia colik-12 and their isolated surface hybrid recombined protein, applied against corona, has not been discussed till date. As the per the devoted, science and life sciences matured intuition, the author on microbes, yeas long day and night keen observations in experiment interest shown in academic maturity emphasized, that "IS-Tn-IS" hybrid, spike protein DNA recombined hybrid GE (Genetically Engineered ) E.coli K-12 hybrid protein, applied orally perhaps would show the relief and safety of mankind.

\section{Literature cited:}

[1]Brahma, N.K., Schumacher, A., Cullum, J. and Saedler H. (1982) Distribution of the Escherichia coli K-12 Insertion Sequences IS1, IS2 and IS3 Among other Bacterial Species. J. Gen. Microbiol. 128: p: 2229-2235.

[2] Brahma, N.K. (1999) Enterobacterial 026: MRHA-plasmid and its possible Genetic Transformation into Auxotrophic Escherichia coli K-12 C600 NS. Indian J. Microbiol. 38 (4): p: 43-50.

[3] Brahma, N.K. (1999) Genetically Engineered Hybrid 5405 E.coli K-12 fimbriae in vaccination With Balb/c mice against 026: serotype EPEC diarrhea. Ind. J.Chem. Engg. 39 (1) p: 17-, Cited in British Journal catalogue (BJC)-UK.

[4] Starlinger P, Saedler H (1976) IS Element in Microorganism; Current Topic on Microbiology and Immunology, 75:, p:111-152,

[5] Ruediger Schmidt (1982),4. Transposon; Springende Gene in Bakterien, Erbforschung Heute. Herausgegeben vom Walter Klingmueller, Verlage Chemie, Basel, Switzerland.

[6] Saedler, H, Ghosal D, and Nevers P, (1978), The role of Gene Rearrangement in Evolution, Institute For Biology III, Schaenzler Strasse, 1;7800 Freiburg, Federal Republic of Germany.

[7]. Maier RM, Pepper IL, Gerba CP,(2009), Environmental Microbiology, (2 ${ }^{\text {nd }}$ Edition), Elsevier, Academic presss.

[8]Retro transposons, July 26, 2018, Source: Carnegie Institution for Science group of U.K.

[9] Jaidip Dogra, Livodip Dogra, 2020, "Opportunistic Chlamydia in Corona, "Pratidin Bengali newspaper, Jaipur Central Govt Health Scheme, $25^{\text {th }}$ May, p:1 -5 .

[10] Braude AI, Davis, CE, Fierer j, (1981) Medical Microbiology and Infectious Diseases, Saunders Pub, International Text Book of Medicine.Page:619, D.A.J.Tyrrell Corona Virus

[11] W. Shang et al. (2020) Vaccines 5, p:18 (2020).http://doi.org/ggrnbr

[12] David A.J. Tyrrell and Steven H. Myint, (1980) Medical Microbiology. 4th editionBottom of Form, https://www.nih.gov/coronavirus

[13]. Dae Gyann Ahn, et.al(2011) Interference of Ribosomal Frame shifting by antisera peptide nucleic acids suppresses SARS ,Corona virus replication, Elsevier 91(1):p:1-10. 
[14] Jei Cui , Fang Li and Zang Li Shi(2018) Origin and Evolution of Pathogenic Coronaviruses, Nature Review Microbiology, 17:p:181-192.

[15] F. Amana \& F. Krammer (2020) Immunity 52:, p:583-589

[16] Felix Boecker and Karin Moelling (2019) Evolution of Immune Systems from Viruses and Transposable Elements. Frontier of Microbiology, Virology, 29 Jan;; CRISPER-Cas a nd RAG $\frac{1}{2}$ T-cell diversity

[17] Mitch Leslie (2020) T-Cell formed in Covid-19, patients "bode cell" for long term Immunity, Science, 14 May.

[18] Bonny Rochx et. al (2020) Comparative Pathogenesis of Covid-19 MERS, SARS in a non-human Primate Model, Science, April.

[19] Amanda Heits, (2020), Giant Viruses are not alive. So why have the stolen gene essential for life, Science, April.

[20] Dominika Hobernik and Matgias Bros, (2018), DNA Vaccine-How Far From Clinical use. Int. J. Mol. Science, 19(11):, 3605 .

[21An updated guide to the coronavirus drugs and vaccines in development By Damian Garde@damiangarde, March 2020 Moderna Therapeutics Approach: Vaccine, ASM Communications

[22]. Thanh Le T, Andreadakis Z, Kumar A, Gómez Román R, Tollefsen S, Saville M, Mayhew S (9 April 2020). "The COVID-19 vaccine development landscape". Nature Reviews Drug Discovery. 19 (5): 305-306.

[23] Jonathan Corum, Knvul Sheikh and Carl Zimmer, May 20, 2020 Different Approaches to a Coronavirus Vaccine

[24] Timothy R. Abbott, Girija Dhamdhere, Yanxia Liu, Xueqiu Lin, Laine Goudy, Leiping Zeng, Augustine Chemparathy, Stephen Chmura, Nicholas S. Heaton, Robert Debs, Tara Pande, Drew Endy, Marie La Russa, David B. Lewis, Qi, Development of CRISPR as a prophylactic strategy to combat novel coronavirus and influenza doi: https://doi.org/10.1101/2020.03.13.991307, Cold Spring Harbor Laboratory,Biorexiv.

[25] Tim Abbott, Could Crispr Be Humanity's Next Virus Killer? Stanford scientists are exploring whether gene-editing technology can be used to fight pandemics. But so far, they have just one piece of a larger puzzle. Science, Stanford University, 2020.

\section{Acknowledgement:}

I thank Prof. Heinz Saedler, who recognized me and my skill of work on Microbiology, allowed me to get involved in his group in Institute of Genetic Engineering (Biology-III Institute, AL-University, Freiburg.) to study Transposable element during 1979-1981 in Germany. I thnak also Dr. John Cullum, a fellow of Royal Society of the same groups, helped me learning genetic engineering of microbial system and DNA hybridizations.

\section{Hosted file}

Figure-AIChE-New-NKB.docx available at https://authorea.com/users/334502/articles/460455understanding-invasive-and-rapid-transmitting-nature-of-corona-through-aair-and-by-is-

tn-is-dna-probe-analysis 